\title{
PSR ELECTRON CLOUD DETECTOR AND SUPPRESSOR MECHANICAL DESIGN AND FABRICATION*
}

\author{
J. F. O’Hara ${ }^{\#, 1}$, R.J. Macek ${ }^{1,2}$, A. A. Browman ${ }^{1,2}$, M. J. Borden ${ }^{1}$, J. E. Ledford ${ }^{1,2}$, N. A. Gillespie ${ }^{1}$, \\ D. Martinez ${ }^{1}$, K. G. McKeown ${ }^{1}$, and F. R. Olivas ${ }^{1}$ \\ ${ }^{1}$ Los Alamos National Laboratory, Los Alamos, NM 87545, U.S.A. \\ ${ }^{2}$ TechSource, Inc., Santa Fe, NM, 87505, U.S.A.
}

\section{Abstract}

In order to better understand the two stream e-p instability issue in the LANSCE Proton Storage Ring (PSR), a new diagnostic instrument has been developed to measure the electron cloud formation and trapping in a quadrupole magnet at the LANSCE PSR. The device called the Electron Cloud Detector (ECD) was fabricated and has successfully been installed in the PSR. Along with the Electron Cloud Detector, an additional device was developed to manipulate electrons ejected from the quadrupole and allow additional information to be obtained from ECD measurements. This paper will discuss the mechanical design and fabrication issues encountered during the course of developing both devices.

\section{ELECTRON CLOUD DETECTOR}

A diagnostic has been developed to study the electron cloud generation and trapping in a PSR quadrupole [1]. The concept is to measure the electron flux that strikes the beam pipe wall during beam induced multipactoring. The device also features an electrode that can be pulsed after the beam pulse has left the magnet to sweep out any electrons that are trapped in the quadrupole. This is a second generation of an electron sweeping device [2] that had been used in drift spaces in the PSR. This new device will reside within a quadrupole in sector four of the PSR. Figure 1 shows the ECD installed in the PSR quadrupole. The existing magnet in that location was removed along with its beam position monitor and a spare quadrupole containing the new ECD was installed.

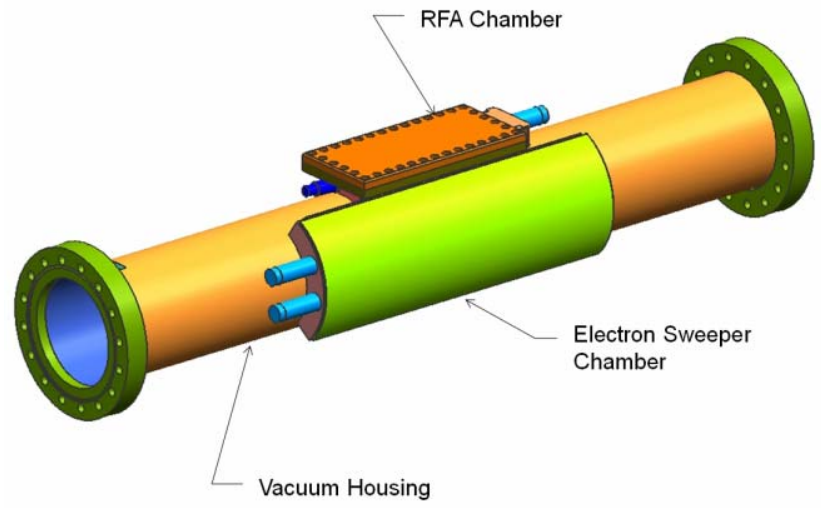

Figure 1: Electron Cloud Detector Beam-line Device.

*Work supported by US Department of Energy SBIR Grant No. DEFG02-04ER84105 and CRADA No. LA05C10535 between

TechSource, Inc. and the Los Alamos National Laboratory

\#ohara@lanl.gov

06 Instrumentation, Controls, Feedback \& Operational Aspects

\section{ECD FEATURES}

The ECD is made up of three main parts, a Retarding Field Analyzer (RFA) [3] chamber, a sweeping electrode, and the vacuum housing. The ECD is oriented within the quadrupole so that the sweeping electrode and the edge of the RFA collector are normal to the quadrupole magnetic field lines. Angular alignment of the ECD in the quadrupole \pm 1 degree was specified. The RFA assembly consists of an electron filter, an electron repeller, and an electron collector. The operating concept is that electrons within the quadrupole will spiral around the quadrupole magnetic field lines and eventually move into the RFA through a series of holes in the beam pipe wall. The repeller grid will have a negative bias applied to it and only electrons of sufficient velocity will be able to pass through and onto the collector where the electron signal is detected.

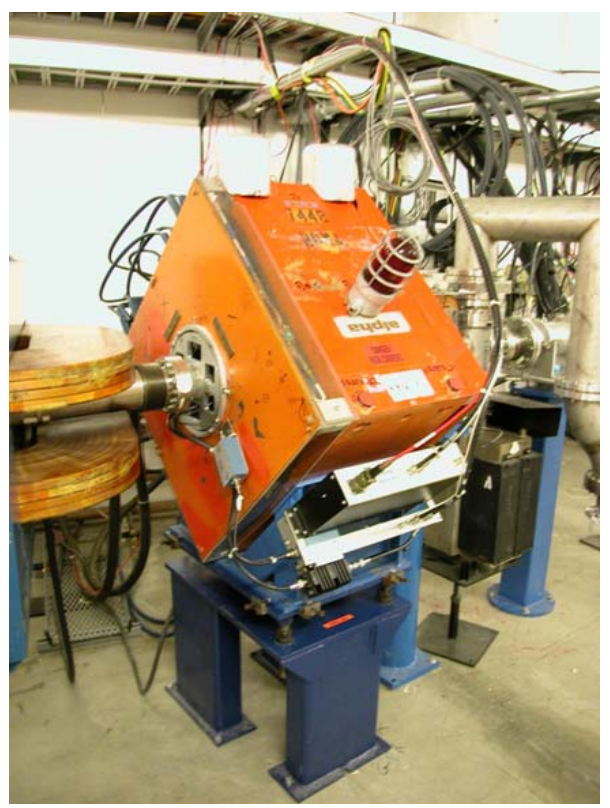

Figure 2: ECD Installed in PSR quadrupole.

The ECD is designed to detect two types of electron signals; prompt electrons and swept electrons. The prompt electrons are those produced by the beam induced multipactoring while the sweeping electrode is grounded. The swept electrons are those that are stay trapped in the quadrupole after the beam has passed through and are then driven into the RFA collector by applying a fast rising voltage pulse of -485 volts to the sweeping electrode.

T03 Beam Diagnostics and Instrumentation 


\section{RFA Assembly}

The RFA assembly is a key part of the ECD device. This assembly resides outside the bore of the beam pipe and within a chamber built into the side of the device by welding a pre-fabricated box to the side of the beam pipe. A slot was cut in the beam pipe with a close tolerance fit (0.000-in to 0.002 -in clearance) around all sides so that the RFA assembly would fit precisely into the beam pipe. This assembly consists of three separate layers where each of the functions of the RFA assembly are performed:

- The first layer is the RFA electron entrance assembly a stainless steel plate with a series of 0.109 -in diameter holes drilled through it. That plate is covered by a copper backing plate with 0.172-in diameter matching holes, which is in turn covered by copper cover plate that has a woven copper screen cloth of 0.002-in diameter copper wire soldered to it. This stainless/copper plate assembly takes the place of the beam pipe wall and the holes are made to allow electrons to pass through unimpeded. The copper plate overlaps the stainless plate and maintains electrical contact to the beam pipe wall. Future test plans include the possibility of changing out this assembly and using one with different size holes to see what effect the different size holes may make to the measurement.

- The second layer is separated from the entrance assembly by a capacitive bar assembly. This next layer is the repeller grid and it consists of a copper plate that also has the same woven copper wire mesh soldered to it. A negative bias is applied to this grid which "filters" out electrons with insufficient velocity. The soldering operation to join the mesh to the plate is done by fluxing the entire surface to be wetted, evenly heating the copper plate and mesh with the use of a "hot plate", and then carefully flowing the solder between the two parts. A lead free solder was used for all the ECD soldering operation. Figure 3 shows the first two layers installed in the ECD, the holes in the beam tube and the repeller grid.

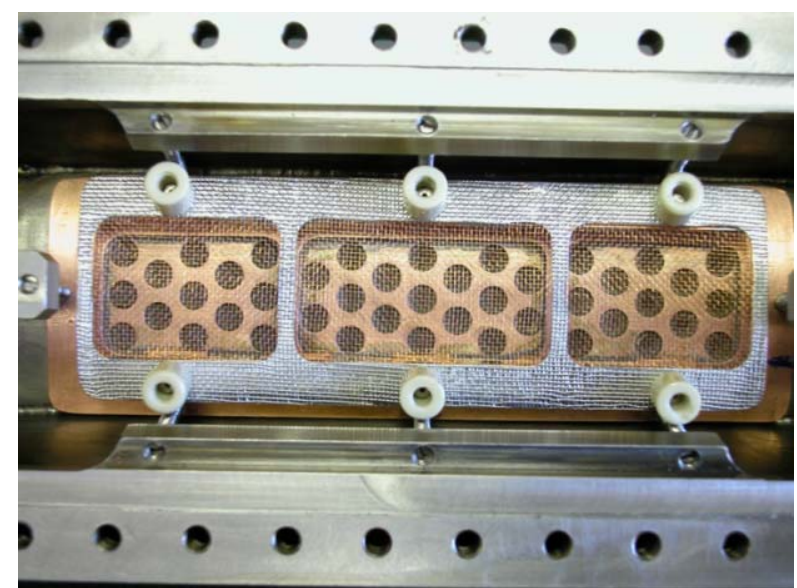

Figure 3: RFA Electron Entrance Assembly and Repeller Grid Installed.

06 Instrumentation, Controls, Feedback \& Operational Aspects
- The final layer is the collector plate which is a copper plate that is fastened to the ceramic insulators that are visible in figure 3. A wire is soldered to this plate that runs to a vacuum feed-through welded into the wall of the RFA chamber. This plate has a +45 Volt bias applied to it. Figure 4 shows the collector plate installed in the ECD. Figure 5 is an exploded view of the RFA Assembly.

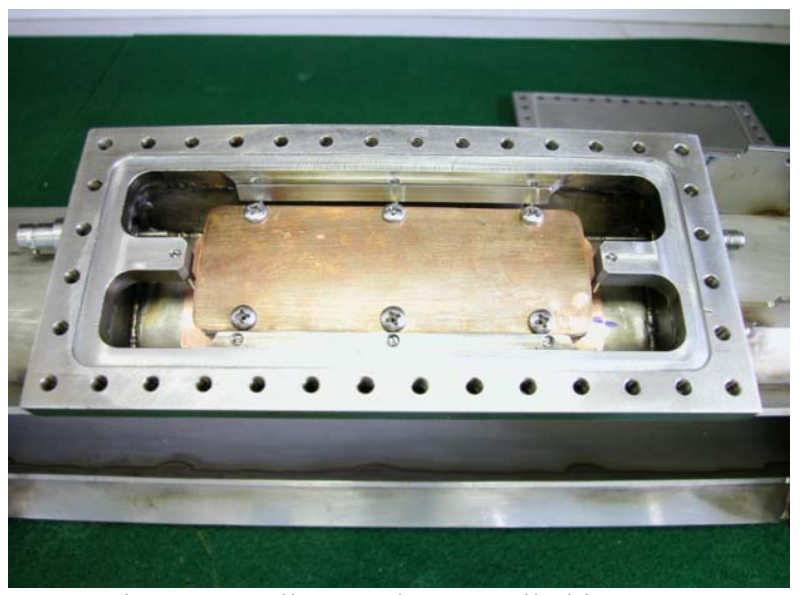

Figure 4: Collector Plate Installed in ECD.

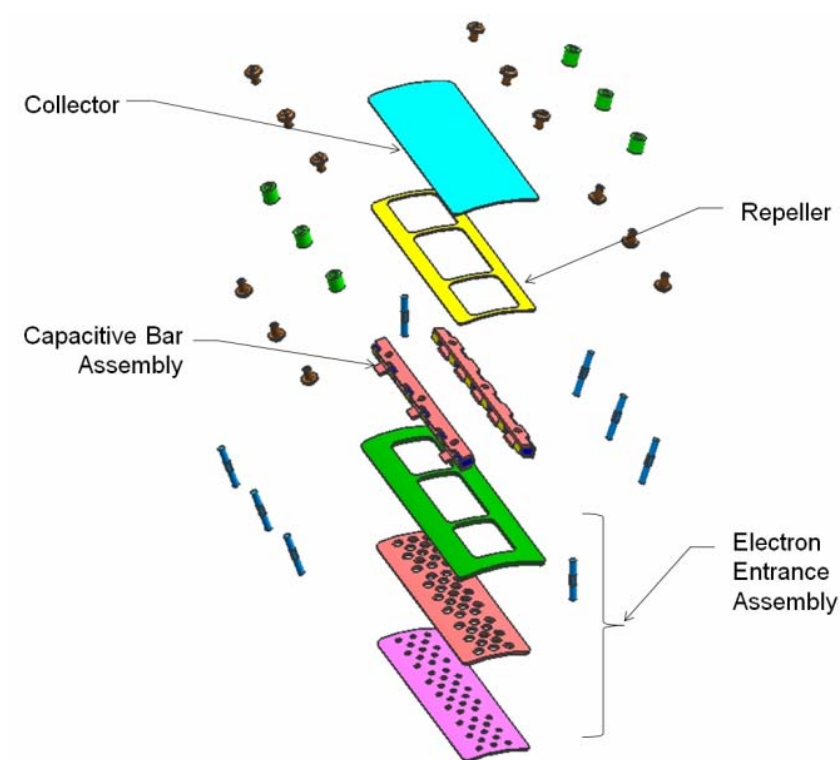

Figure 5: Exploded View of the RFA Assembly.

\section{Electron Sweeper}

The electron sweeper assembly consists of another welded chamber on the ECD beam pipe located 81degrees away from the center of the RFA assembly. Another slot is machined out of the beam pipe and a stainless steel plate having the same inside radius as the beam tube is mounted in the slot so that the desired clearance of 0.080 -inches all the way around is achieved. The plate is mounted to isolating ceramic stand-offs that are in turn attached to stainless steel welding tabs. Belleville washers were used between the ceramic and weld tab to provide needed compliance which prevented cracking of the ceramic standoff when the screw was

T03 Beam Diagnostics and Instrumentation 
tightened. The welding tabs are designed so that they can be machined to achieve the evenly distributed clearance desired. The tabs are then "tack" welded to the sweeper chamber wall. Two connections for the application of the -485 Volt fast rising pulse are soldered to vacuum feedthroughs. Figure 6 shows the installed electron sweeper plate with the sweeper chamber cover removed.

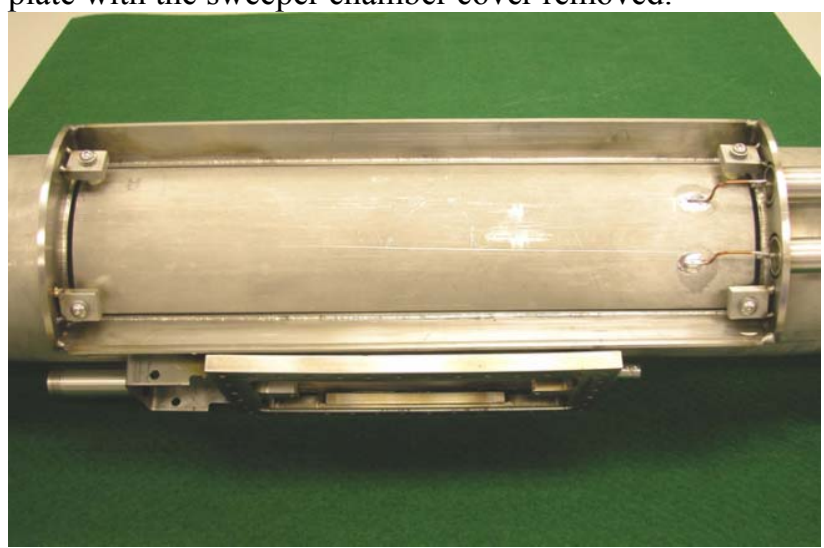

Figure 6: Electron Sweeper Plate Installed in ECD.

\section{ECD Vacuum Housing}

The vacuum housing is constructed of a series of weldments joined to a stainless steel beam pipe. The stainless steel material used was 316 full solution annealed. Two cut-outs were machined into the wall of the standard 4-inch diameter beam pipe to accommodate the RFA and electron sweeper assemblies. The chambers for these two pieces were made from plates of the same 316 stainless steel welded together using 310-ER welding rod or joined using fusion welds. Maintaining the desired fit between the beam pipe wall and the RFA entrance plate of 0.000 -in to 0.002 -in was a challenge. The other requirement for this fit was that the surface of the RFA entrance plate had to be flush with the inner radius of the beam pipe. The entrance plate was machined to the specified tolerances and the slot in the beam pipe was custom machined to achieve the desired fit.

Since the ECD resides with a quadrupole another requirement was to make the welded joints with a magnetic permeability, $\mu$, as close to 1.0 as possible so as to minimize any disturbance of the quadrupole fields. The 316 stainless welded with the 310 rod has been shown to produce welds having a permeability of between 1.02 and 1.05 [4]. This level of permeability was determined to be acceptable. In fact the ECD welds were measured to be within this range. The location of highest permeability, between 1.05 and 1.10 , was found in the region near the tapped holes in the vacuum flange of the RFA chamber.

\section{ELECTRON MIRROR}

A second device has been built to further help understand the electron cloud generation. This device acts as a barrier to electrons moving along the beam axis and has been called an electron "mirror" [5]. Figure 7 shows a cross section of the device. It consists of a nominal 6-inch beam tube vacuum chamber housing a 4inch diameter by $30 \mathrm{~cm}$ long cylinder. This bias ring is isolated from the outer vacuum chamber by use of macor support rings. High voltage feed-throughs and connecting wires are used to apply the bias voltage to the plate.

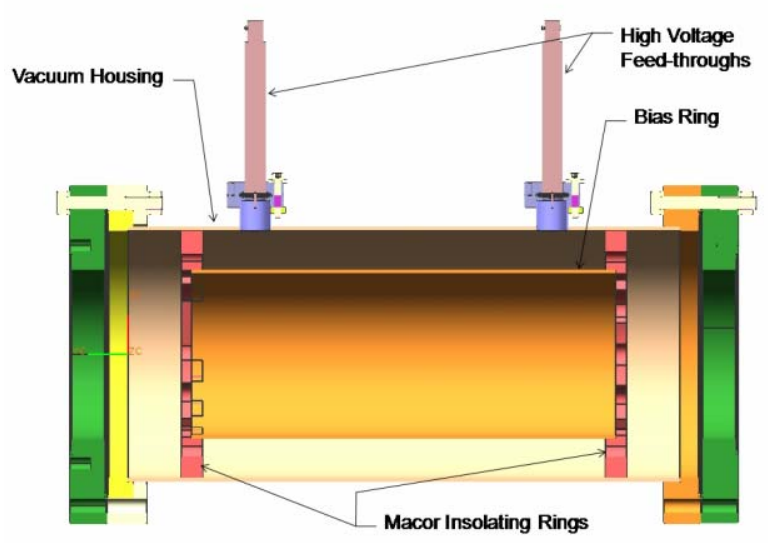

Figure 7: Electron Mirror Cross Section.

One of these Electron Mirror devices was installed in section 4 of the PSR prior to the extended outage in December of 2006 and some testing was accomplished. A second device was fabricated and installed during the extended outage and further tests with the second device will be conducted during the up-coming LANSCE run cycle.

\section{REFERENCES}

[1] Macek, R. J. et al., "Electron Cloud Generation and Trapping in a Quadrupole Magnet at the Los Alamos PSR", to be published at the 2007 Particle Accelerator Conference, Albuquerque, NM, June 2529, 2007.

[2] Macek, R. J. et al., "Electron Cloud Diagnostics in Use at the Los Alamos PSR", Proceedings of the 2003 Particle Accelerator Conference, p. 508 (2003)

[3] R. A. Rosenberg and K. C. Harkay, "A Rudimentary Electron Energy Analyzer for Accelerator Diagnostics", NIM A453, p. 507 (2000).

[4] N. Wilson and P. Bunch, "Magnetic Permeability of Stainless Steel For Use in Accelerator Beam Transport Systems", Proceedings of the 1991 IEEE Particle Accelerator Conference, San Francisco, CA, May 6-9, 1991.

[5] Macek, R. J. et al., "Electron Cloud Generation and Trapping in a Quadrupole Magnet at the Los Alamos PSR", to be published in the proceedings of the ECLOUD’07 Workshop, April 9-12, 2007 Daegu, Korea. 